\title{
Rheumatoid arthritis: effects of a new agent (ICI 55 897) on serum acute phase proteins and the erythrocyte sedimentation rate
}

\author{
B. MCCONKEY, ${ }^{1}$ R. S. AMOS, ${ }^{1}$ M. E. J. BILLINGHAM, ${ }^{2}$ \\ T. J. CONSTABLE, 1 R. A. CROCKSON,, 3 . P. CROCKSON, ${ }^{3}$ \\ AND P. J. G. FORSTER ${ }^{1}$
}

From the ${ }^{1}$ Dudley Road Hospital, Birmingham, B18 7QH, the ${ }^{3}$ Department of Experimental Pathology, Birmingham University, and ${ }^{2}$ ICI Pharmaceutical Division, Macclesfield

SUMMARY Thirty-four patients with rheumatoid arthritis (RA) were treated with a new agent (ICI 55897 ) in addition to basic therapy with nonsteroid anti-inflammatory drugs. Five patients had the drug for less than 28 days; the remaining 29 were observed for periods up to a year. At 140 days, when all but 2 patients were in the study, there had been statistically significant improvement in clinical score, serum C-reactive protein, erythrocyte sedimentation rate, and plasma fibrinogen. Thereafter results continued to improve but were biased because some patients had stopped taking the drug. The final conclusion was that 17 patients had improved with 1 late relapse, and 15 had not responded. Adverse effects were trivial except in 2 instances: one patient had a transient unexplained rise in blood urea, another had a haematemesis. Neither effect could be attributed with certainty to the drug. ICI 55897 has no intrinsic analgesic or anti-inflammatory properties. We suggest the findings of this study indicate that this agent, with low toxicity and the ability to lower acute-phase protein levels, may be an alternative to gold or penicillamine in the treatment of RA.

A vital characteristic of any successful drug for rheumatoid arthritis (RA) must be its ability to relieve pain. This alone, however, is not enough. Any painful disorder might be temporarily alleviated by a drug whose principal effect was analgesic and/or anti-inflammatory, but it would not follow that the underlying disease process had been influenced. The additional property required of a drug for RA is the ability to control the disease. We have previously suggested that this property can be inferred when a drug, for example gold, influences the levels of the serum acute phase proteins (APP) and the erythrocyte sedimentation rate (ESR) (McConkey et al., 1972, 1973; Amos et al., 1977); moreover the duration and magnitude of this effect provide a guide to the efficacy of a drug (McConkey et al., 1979).

In this paper we report a study, based on measurements of APP and ESR, of a new agent (ICI 55 897) in RA. This drug had been found to inhibit a chronic form of arthritis in the rat (Billingham and

Accepted for publication 23 February 1979

Correspondence to Dr McConkey.
Davies, 1978). It possessed minimal activity in models of acute inflammation, was not analgesic, and thus was considered to possess a novel spectrum of pharmacological activity compared to nonsteroidal anti-inflammatory drugs (NSAID). ICI 55897 is a substituted benzyloxy-methyl propionic acid, thus quite distinct chemically as well as biologically from the aryl propionic acids of the NSAID group.

\section{Patients and methods}

We studied 34 patients with classical or definite RA (American Rheumatism Association's criteria). Five of them stopped the drug after less than 28 days and are excluded hereafter except in the description of adverse effects. The remaining 20 women and 9 men had a mean age of 58.4 years (range 27 to 76 years) and mean duration of RA 11.4 years (range 2 to 32 years). Nineteen patients had a positive Rose-Waaler test.

No patient was receiving gold, dapsone, penicillamine, antimalarials, corticosteroids, or cytotoxic 
agents during the study, but all continued to take a NSAID, usually indomethacin. After a preliminary assessment period of at least 28 days ICI 55897 was started at a dose of $50 \mathrm{mg}$ daily, increasing by stages to $200 \mathrm{mg}$ daily in a single dose.

The subjective clinical state was recorded as clinical score (McConkey et al., 1972). At the first visit each patient was allotted an arbitrary clinical score of 100 . Subsequently, at every visit the patient was asked 'Do you feel better, worse, or the same compared to last time?' The score of 100 was altered by +2 if better, -2 if worse. Serum C-reactive protein (CRP) was measured by radial immunodiffusion, serum haptoglobin $(\mathrm{Hp})$ as haemoglobin binding capacity (Ratcliff and Hardwicke, 1964), ESR by the Westergren method, and plasma fibrinogen by nephalometry.

The clinical score at the start of treatment was standardised to 100 . Because patients did not attend at exactly regular intervals the data were grouped in intervals of $28 \pm 14$ days. Comparisons were made between mean values for the observations before and after treatment began, and Wilcoxon's rank sum test was used to analyse the results.

\section{Results}

Table 1 shows the mean values for the clinical score and the 4 measurements (CRP, Hp, fibrinogen, and ESR) $28 \pm 14$ days before treatment, at the start of treatment, and at intervals thereafter. There were no significant differences between the 2 sets of pretreatment observations. After treatment began there were progressive trends towards improvement in clinical score and in the measurements (Table 1).

At 140 days all but 2 patients, who had not responded, were still in the study; by that time there had been a rise in clinical score and falls in the mean values for CRP, ESR, and fibrinogen. Results for CRP (Fig. 1) illustrate the change in the distribution of the data. Moreover, at the start of the trial each patient had a serum CRP $>20 \mathrm{mg} / \mathrm{l}$, whereas at 140 days 10 patients had a serum CRP $<20 \mathrm{mg} / \mathrm{l}$. Results for ESR and fibrinogen were similar. The falls in serum Hp were smaller and did not reach statistical significance until later.

Between 140 and 364 days 16 patients stopped taking the drug (Fig. 2). Nine of them had not responded; 6 were better, as indicated by a sustained rise in clinical score with a fall in CRP to $<20 \mathrm{mg} / \mathrm{l}$ and ESR to $<30 \mathrm{~mm} / \mathrm{h}$; and 1 had a haematemesis.

One other patient had just completed 140 days' treatment at the time of analysis of the data. The remaining 10 patients were better at 140 days. (CRP

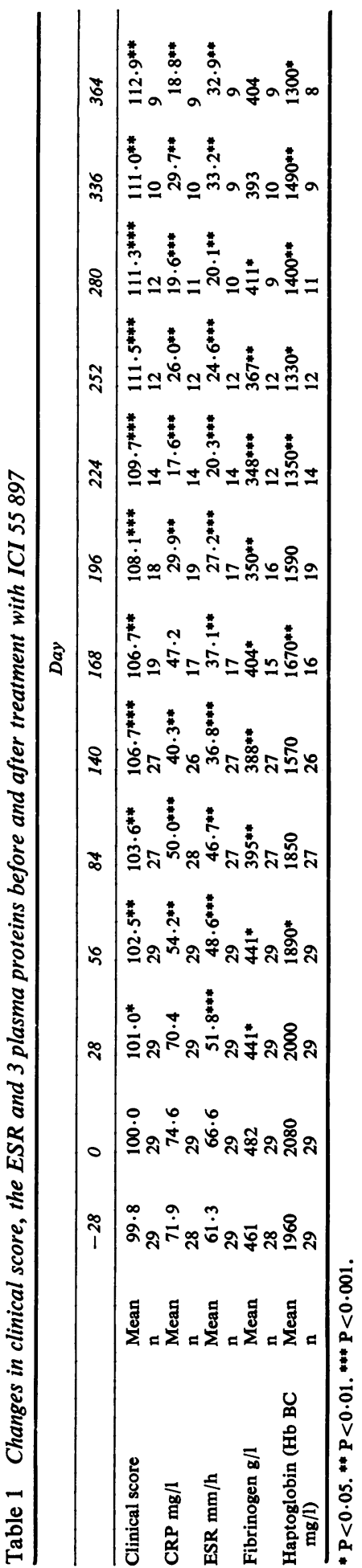




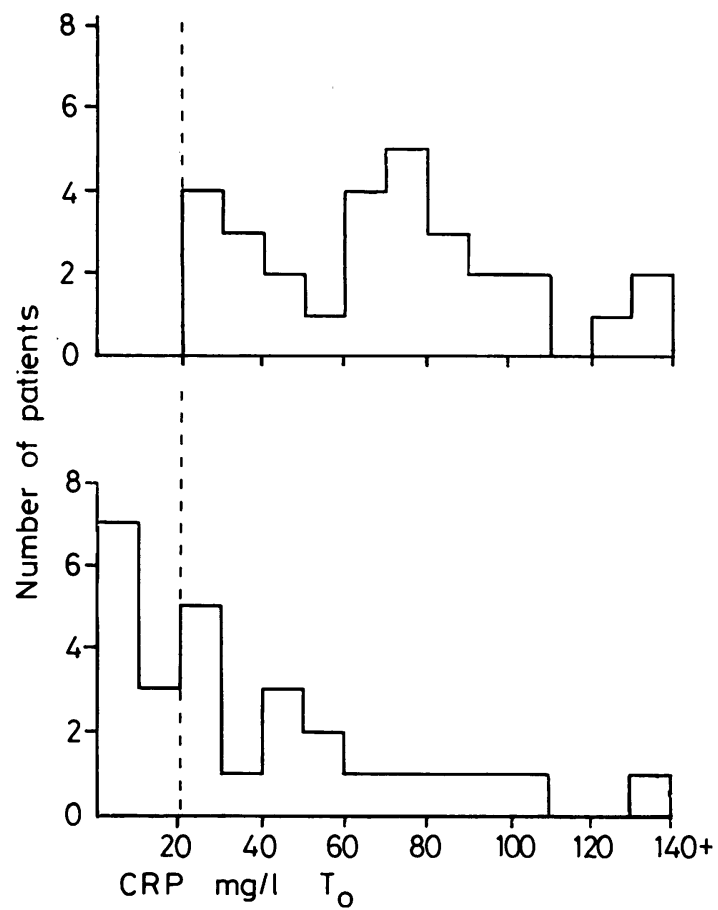

Fig. 1 Distribution of values for serum CRP at the start of treatment $\left(T_{\mathrm{o}}\right)$ with ICI 55897 and at 140 days $\left(T_{140}\right)$ in 27 patients

Initial recruitment

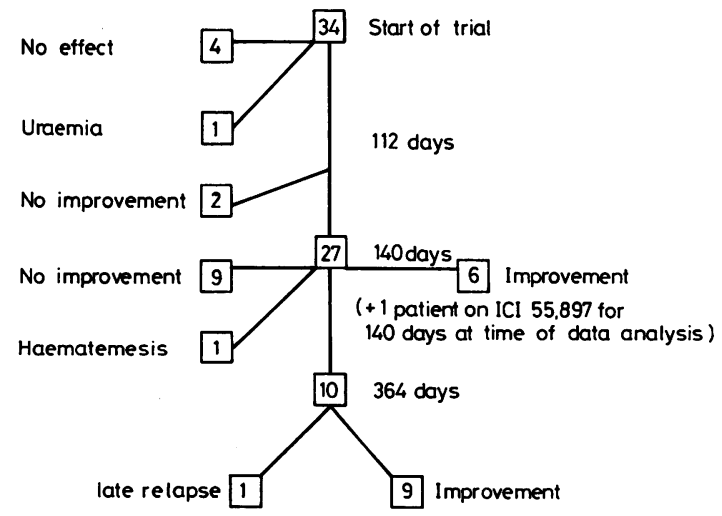

Fig. 2 Outcome of 34 patients in trial of ICI 55897

$<20 \mathrm{mg} / \mathrm{l}, \mathrm{ESR}<30 \mathrm{~mm} / \mathrm{h}$ ) and continued treatment to the end of the period of study, 364 days; at that time 1 of them showed signs of relapse.

There was no significant change in RW titre.
ADVERSE EFFECTS

Five patients stopped the drug after less than 28 days, 4 of them because of lack of effect and 1 because of an unexplained rise in serum urea. No other biochemical or haematological abnormalities were observed. Side effects were uncommon, being vague dyspepsia nct necessitating withdrawal of the drug. The patient who had a haematemesis was investigated for the cause of the bleeding with negative results. He was assumed to have had an acute gastric erosion, and treatment with ICI 55897 was restarted in a later trial without any recurrence of gastrointestinal disturbance.

\section{Discussion}

We have previously shown that nonsteroid antiinflammatory drugs do not influence the levels of serum APP or the ESR (McConkey et al., 1973; Amos et al., 1978). Low levels of these measurements are associated with a slower rate of progression of RA judged by bone erosions on $x$-rays (Amos et al., 1977). Thus drugs such as gold, which lower APP levels (McConkey et al., 1976, 1979), might be expected to slow the progress of the disease if their effect is exerted for sufficient time.

The results of this study show that in a group ofo 29 patients who were in a steady state for at least a month before starting treatment ICI 55897 led to significant improvement in subjective clinical state and significant falls in serum CRP, serum Hp, ESR, and plasma fibrinogen. The effect was small at 28 days but substantial by 56 days, thereafter increasing. At 140 days, when all but 2 patients were still in the study, values for CRP and ESR had fallen in a third of the patients to levels which we regard as acceptable based on a study of $x$-ray data (Amos et al., 1978). After that time results are biased by falling numbers of patients. The bias is hard to assess because in the time interval 140-364 days one-third of the patients stopped the drug because they were better, two-thirds for lack of response. The effect of this reduction in numbers probably makes the results between 140 and 364 days seem better than they are. However, at 1 year 10 patients were still taking ICI 55897 ; 9 of them were in almost complete remission and 1 showed evidence of relapse. Comparison of the falls in the 4 measurements suggests they were not of equivalent magnitude; the falls in CRP and ESR exceeded those in fibrinogen and $\mathrm{Hp}$. The clinical significance of this finding is unknown but we have shown (McConkey et al., 1979) that prednisone has a greater effect on CRP than on $\mathrm{Hp}$, whereas gold affects both similarly.

The results suggest that ICI 55897 may be a useful addition to the range of drugs used to treat 
RA. Its place is likely to be as an alternative to gold or penicillamine, though it does not appear to lower rheumatoid factor levels. One important advantage is its apparent low toxicity in animal experiments and in this clinical trial. The drug is still in the clinical trial phase.

This work was supported by the West Midlands Regional Health Authority and by Imperial Chemical Industries Ltd. The statistical calculations were done by Miss E. A. Laws, ICI Pharmaceuticals Division.

\section{References}

Amos, R. S., Constable, T. J., Crockson, R. A., Crockson, A. P., and McConkey, B. (1977). Rheumatoid arthritis: relation of serum $C$-reactive protein and erythrocyte sedimentation rates to radiographic changes. British Medical Journal, 1, 195-197.

Amos, R. S., Crockson, R. A., Crockson, A. P., Walsh, L., and McConkey, B. (1978). Rheumatoid arthritis: Creactive protein and erythrocyte sedimentation rate during initial treatment. British Medical Journal, 1, 1396-1397.
Billingham, M. E. J., and Davies, G. E. (1978). In Inflammation. Handbook of Experimental Pharmacology, 50, chapter 23. Edited by J. Vane and S. H. Ferriera. Springer Verlag: Berlin.

McConkey, B., Crockson, R. A., and Crockson, A. P. (1972). Assessment of rheumatoid arthritis: a study based on measurements of the acute phase reactants. Quarterly Journal of Medicine, 41, 115-125.

McConkey, B., Crockson, R. A., Crockson, A. P., and Wilkinson, A. R. (1973). The effects of some anti-inflammatory drugs on the acute phase proteins in rheumatoid arthritis. Quarterly Journal of Medicine, 42, 785-791.

McConkey, B., Davies, P., Crockson, R. A., Crockson, A. P., Butler, M., and Constable, T. J. (1976). Dapsone in rheumatoid arthritis. Rheumatology and Rehabilitation, 15, 230-234.

McConkey, B., Davies, P., Crockson, R. A., Crockson, A. P., Butler, M., Constable, T. J., and Amos, R. S. (1979). Effects of gold, dapsone, and prednisone on serum Creactive protein and haptoglobin and ESR in rheumatoid arthritis. Annals of the Rheumatic Diseases, 38, 141-144.

Ratcliff, A. P., and Hardwicke, J. (1964). Estimation of serum haemoglobin-binding capacity (haptoglobin) on Sephadex G 100. Journal of Clinical Pathology, 17, 676679. 